\title{
OPEN Strain-dependent structure and Raman behaviours in the heavy-ion irradiated manganite at extreme low dose
}

\author{
Nam Nhat Hoang ${ }^{1 *}$, Duc Huyen Yen Pham ${ }^{1,2} \&$ The Nghia Nguyen ${ }^{3}$ \\ The microstrains in heavy-ion irradiated manganite $\mathrm{LaMnO}_{3}$ can be managed in linear response of \\ irradiation dose, and the corresponding internal pressure up to $8 \mathrm{GPa}$ can be induced by varying doses. \\ The response of structure under stress is studied by means of Density Functional Theory and Lattice \\ Dynamic Calculation. All obtained Raman scattering lines are discussed in details to shed light onto \\ structural changes during ion implantation. There appears new resonance peak at around $550 \mathrm{~cm}^{-1}$, \\ which splits from broad features in the spectra, and attributes to the anti-symmetric vibrations of $\mathrm{O}_{6}$ \\ cages. The blue shift of this peak scales to $2.4 \mathrm{~cm}^{-1}$ per $1 \mathrm{GPa}$ of stress. Another strong feature showing \\ considerable blue shift is seen in the vicinity of $640 \mathrm{~cm}^{-1}$ and corresponds to one of rhombohedral \\ distortion related soft modes. A weak mode, not frequently reported, is seen at around $420 \mathrm{~cm}^{-1}$ and \\ corresponds to translation-like motions of fixed $\mathrm{O}_{6}$ cages.
}

The $\mathrm{LaMnO}_{3}$ (LMO) has attracted the attention of scientists for both fundamental and application researches for decades due to a number of important effects that coexist within a single structure frame. Among the most interesting ones are colossal magnetoresistance ${ }^{1}$, magnetic and orbital orderings ${ }^{2,3}$, insulator-metal transition and ferromagnetism ${ }^{4,5}$. Many of these are believed to have their origins in the double exchange interaction between $\mathrm{Mn}^{3+}-\mathrm{O}-\mathrm{Mn}^{4+}$ pairs, which is accompanied by Jahn-Teller distortion of coordination octahedra of Mn ions ${ }^{6}$. Although the nominal oxidation state of $\mathrm{Mn}$ in LMO is $3+\left(\right.$ electronic configuration $t_{2 g}^{3} e_{g}^{1}$ ) there always exists a portion of $\mathrm{Mn}$ in $4+$ state $\left(t_{2 g}^{3} e_{g}^{0}\right)$ due to non-stoichiometric oxygen content often presented, so the ferromagnetic double exchange interaction is a typical phenomenon in this compound. It is important to note here that the strength of Jahn-Teller gap $(\approx 2 \mathrm{eV})$ falls within a range of energy covered by Raman resonances, therefore the Raman spectroscopy appears essential for the study of effects arising from magnetic orderings in LMO. Indeed, the early studies ${ }^{2,7}$ have proposed that the orbital orderings may be observed in Raman at a few hundreds $\mathrm{cm}^{-1}$. The first classification of Raman scattering bands for LMO in orthorhombic space group Pnma (no. 62) was given quite early ${ }^{8}$, and later the same group of authors also reported the classification for the higher symmetry $R \overline{3} c$ (no. $167)^{9}$. Basically, LMO exhibits two different structures depending on sintering temperature: the low temperature orthorhombic Pnma and the high temperature rhombohedral $R \overline{3} c$. Both are derived from parent cubic lattice $P m \overline{3}$ $m$ (no. 221). The Raman spectra of these two structures are very similar and possess broad features near 300, 500 and $600 \mathrm{~cm}^{-1}$, but the assignments of modes in the two cases are different. By measuring the Raman scatterings in different orientations and varying temperature (in some cases from below Curie temperature $T_{C}$ to above Néel temperature $T_{N}$ ) the origins of each scattering bands were identified ${ }^{8-11}$. Some high frequency bands (above $1000 \mathrm{~cm}^{-1}$ ) were assigned to orbitons ${ }^{12,13}$ (that is, the excitations of orbital wave according to Franck-Condon mechanism ${ }^{14}$ ) and were claimed as the first experimental observation of orbitons. But later on they were shown to be the false assignments, as the features are originated in multi-phonons ${ }^{15,16}$. The importance of Raman spectroscopy in the study of LMO arises from its high sensitivity to local disorders caused by Jahn-Teller distortions of $\mathrm{MnO}_{6}$ octahedra. It is known that under high pressure the $\mathrm{O}_{6}$ cages can rotate in precession motions against

${ }^{1}$ Laboratory of Low Dimensional Materials and Applications, Faculty of Engineering Physics and Nanotechnology, VNU-University of Engineering and Technology, 144 Xuan Thuy, Cau Giay, Ha Noi, 10000, Viet Nam. ${ }^{2}$ Department of Physics, Chungbuk National University, Cheongju, 28644, South Korea. ${ }^{3}$ Department of Nuclear Physics, Faculty of Physics, VNU-University of Science, 334 Nguyen Trai, Thanh Xuan, Ha Noi, 10000, Viet Nam. *email: namnhat@ gmail.com 


\begin{tabular}{|c|c|c|c|c|c|c|c|c|c|}
\hline Sample & $\begin{array}{l}\mathrm{LaO}^{-} \\
(\mu \mathrm{C})\end{array}$ & $a(\AA)$ & $c(\AA)$ & $c / a$ & $\left|\gamma-90^{\circ}\right| / 90^{\circ}(\%)$ & $x(0)$ & $\mathrm{Mn}-\mathrm{O}_{6}(\AA)$ & $\mathrm{Mn}-\mathrm{O}_{6}-\mathrm{Mn}\left({ }^{\circ}\right)$ & $R_{\mathrm{P}}(\%)$ \\
\hline \multirow{6}{*}{ As prepared } & 0 & $5.512(5)$ & $13.322(4)$ & 2.417 & 0.80 & 0.4521 & 1.958 & 164.5 & 6.3 \\
\hline & 20 & $5.521(4)$ & $13.354(3)$ & 2.419 & 0.76 & 0.4497 & 1.967 & 163.4 & 5.9 \\
\hline & 40 & $5.524(1)$ & $13.367(5)$ & 2.420 & 0.72 & 0.4452 & 1.969 & 162.3 & 5.6 \\
\hline & 60 & $5.528(4)$ & $13.388(4)$ & 2.422 & 0.68 & 0.4436 & 1.972 & 161.8 & 5.8 \\
\hline & 80 & $5.531(1)$ & $13.398(3)$ & 2.422 & 0.67 & 0.4422 & 1.974 & 161.3 & 6.0 \\
\hline & 100 & $5.534(1)$ & $13.395(5)$ & 2.420 & 0.71 & 0.4400 & 1.977 & 160.6 & 5.3 \\
\hline \multirow{5}{*}{ Sintered } & 20 & $5.517(2)$ & $13.331(1)$ & 2.416 & 0.82 & 0.4431 & 1.969 & 161.6 & 5.3 \\
\hline & 40 & $5.519(5)$ & $13.329(8)$ & 2.415 & 0.86 & 0.4440 & 1.967 & 161.9 & 6.5 \\
\hline & 60 & $5.516(7)$ & $13.325(4)$ & 2.416 & 0.83 & 0.4452 & 1.965 & 162.2 & 6.1 \\
\hline & 80 & $5.520(7)$ & $13.333(8)$ & 2.415 & 0.83 & 0.4416 & 1.969 & 161.2 & 6.6 \\
\hline & 100 & \begin{tabular}{|l|}
$5.521(5)$ \\
\end{tabular} & $13.341(8)$ & 2.416 & 0.82 & 0.4420 & 1.968 & 161.3 & 5.2 \\
\hline
\end{tabular}

Table 1. The results of Rietveld refinements for irradiated LMO samples $\left(R \overline{3} c\right.$, no. $\left.167, \alpha=\beta=90^{\circ}, \gamma=120^{\circ}\right)$. The standard deviations are given in the parenthesis, the figure-of-merit $R_{P}$ is for the profiles. The occupation factors of La were all refined to 1.00 , whereas that of $M n$ to 0.99 and $\mathrm{O}$ to 0.98 . The concentrations are given in the total charge of irradiated ions $(\mu \mathrm{C})$. All six $\mathrm{Mn}-\mathrm{O}_{6}$ bond lengths are equal.

each other, promoting the changes in $\mathrm{Mn}-\mathrm{O}$ bond lengths and angles that can be probed by Raman. Indeed, there are a number of LMO studies involving Raman as an analytical tool ${ }^{17-20}$, especially where doping concentrations are low ${ }^{10,21}$. Since the internal pressure can be induced by lattice strains, it is reasonable to question whether or not the strains can be engineered (at ambient pressure) and observed simultaneously for LMO. In this paper we show that the microstrains can be smoothly varied by heavy-ions bombardments, so that the internal pressure is linearly induced, and observed by both X-Ray and Raman measurements. To rule out the impacts associated with changing oxygen stoichiometry we are working only in a region of concentrations that are much lower than the usual oxygen non-stoichiometric content. Our LMO samples are irradiated at extremely low doses by $\mathrm{LaO}^{-} \mathrm{high}$ energy ion beam, resulting in deeply pierced materials of certain density of doped sites. The focus is paid on how the stress created by heavy-ion insertion influences the structure and Raman behaviours of the compounds.

\section{Results and Discussion}

Structure under stress. Because $\mathrm{LaO}^{-}$doses are low $(<0.05 \%$ of La-content in bulk samples), the associated oxygen non-stoichiometry should be minimal. So, we assume that any non-stoichiometric content should be located at the interstitial sites and contributed to the strains. The Rietveld refinements were carried out for all samples in $R \overline{3} c$ space group with optimization for the following variables: lattice parameters $(a, c)$, site occupation factors ( $\mathrm{La}, \mathrm{Mn}, \mathrm{O}$ ), thermal motions, $x$ coordinates of oxygen atoms. Corrections for backgrounds, preferred orientations, zero points were applied. For the determination of crystallite sizes and lattice strains the modified Thompson-Cox-Hastings profiles ${ }^{22}$ were used. The obtained results are summarized in Table 1 . As the typical diffractogram, the one obtained for the as-prepared $40 \mu \mathrm{C}$ irradiated sample is shown in Fig. 1(a), together with the inset which enlarges the strongest reflection before and after sintering for another sample $(100 \mu \mathrm{C})$. It is obvious that there is a shoulder split from the strongest peak at around $32.78^{\circ}$, which is present in all as-prepared samples (Fig. 1(b)) and grows stronger in the sintered ones (Fig. $1(\mathrm{c})$ ). This peak is a typical $R \overline{3} c$ scattering line (014) and is neither present in the higher $P m \overline{3} m$ nor in the lower Pnma symmetry, whereas the strongest peak (around $32.45^{\circ}$ ), which is indexed as (110) in $R \overline{3} c$, is also seen as (011) in $P m \overline{3} m$ or as (200) in $P n m a^{23}$. For the as-prepared samples, Fig. 1(b) depicts two main effects as irradiation increases: the weakening of $R \overline{3} c$-related (014) shoulder reflections and the clear shift to lower angles of (110) and (014) peaks. While the first argues for shifts of symmetry towards lower Pnma at higher irradiation doses, the second implies increases of lattice constants as dose increases. Table 1 shows that, there is a systematic prolongation of all axes, but this seems to be an isotropic effect with no preferred orientation as the $c / a$ ratios do not change convincingly from a value of the un-doped sample. The average increase of unit cell volumes is about $1 \%$ (the largest increase is $1.35 \%$ for $100 \mu \mathrm{C}$ sample). On the other hand, Fig. 1(c) does not show the similar changes for sintered samples, indeed the lattice parameters of all sintered samples are very close to a value of the un-doped one. A clear relaxation of lattice after sintering can also be seen in the inset of Fig. 1(a). To clarify this relaxation, Fig. 2(a) compares the lattice parameters of the as-prepared and the sintered samples. It is evident that after increases during irradiation the lattices of sintered samples compressed and relaxed to the smaller lattices, close to that of the un-doped structure. Let us consider the changes of symmetry by converting the $R \overline{3} c$ cells into the pseudo-Pnma by using a transformation matrix $[(1,2 / 3,-1 / 3) ;(0,4 / 3,-2 / 3) ;(0,1 / 3,1 / 3)]$. Doing so, we obtain the Pnma axes of appropriate lengths, but they lose orthogonality: $\gamma \approx 90^{\circ}\left(\alpha=\beta=90^{\circ}\right)$. As seen in Table 1 , the deviations of $\gamma$ from $90^{\circ}$ are equal for the un-doped and sintered samples (this means they remain $R \overline{3} c$ ) but visibly show a decrease for the as-prepared samples, which implies shifts of symmetry towards the lower Pnma.

The increase of unit cell volumes after irradiation is not usual but may be understood as a consequence of loading (or unloading) internal (or external) pressure. The supposed lattice strains can be directly obtained from relative increases of lattice constants $\varepsilon_{a}=\Delta a / a, \varepsilon_{\mathrm{c}}=\Delta c / c$. For the as-prepared samples the average $\varepsilon_{a}$ and $\varepsilon_{c}$ are 0.24 and $0.37 \%$, and for the sintered ones they reduce to 0.12 and $0.07 \%$ correspondingly. This decrease agrees well with observed relaxations after heat treatment. It is evident that, $\varepsilon_{a}$ and $\varepsilon_{c}$ themselves are not adequate in 

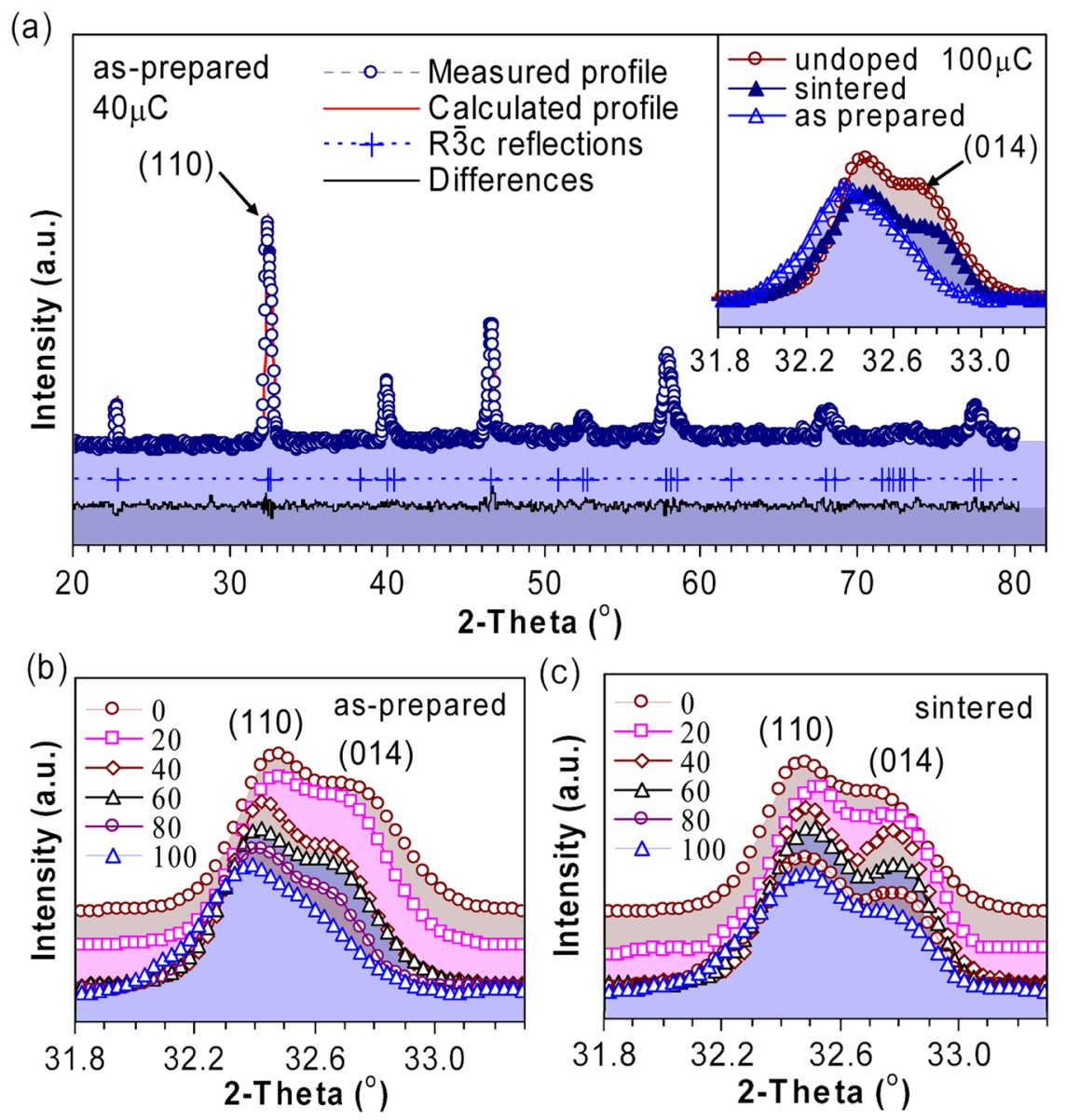

Figure 1. X-ray diffractograms of irradiated samples. (a) The measured and calculated profiles for as-prepared $40 \mu \mathrm{C}$ irradiated sample; the inset compares the profiles of the strongest reflection before and after sintering for $100 \mu \mathrm{C}$ sample. The profiles of the strongest reflection are shown for (b) as-prepared and (c) sintered samples.

describing the total internal stress, as the individual lattices, including the un-doped one, are bearing their own non-uniform microstrains which attribute to the broadening $\Delta \theta$ of diffraction lines. Putting aside the irrelevant (instrumental and crystallite size broadening), in quasi-cubic lattices the broadening caused by lattice strains is linear function of $\tan \theta_{h k l}$, i.e. $\Delta\left(2 \theta_{h k l}\right)=\varepsilon_{\theta} \tan \theta_{h k l}^{24}$ (assuming the Gaussian peak shape). For the analysis of profile by Rietveld technique, this type of broadening is included in the modified Thompson-Cox-Hastings (TCH) profile $^{22}$. Reasonably, for the samples prepared under the same conditions the average broadening $\varepsilon_{\theta}$ should be close to each other. Indeed, we obtained $0.40 \%$ for the as-prepared, and $0.31 \%$ for the sintered and un-doped samples. The similar trend and close values of microstrains are often seen in the heavy-ion irradiated samples, e.g. in the $\mathrm{Ag}^{9+}$ irradiated $\mathrm{SrTiO}_{3}{ }^{25}$. Thus, the total lattice strain should be given as $\varepsilon=\varepsilon_{a}+\varepsilon_{\theta}$. The obtained results for $\varepsilon$ are shown in Fig. 2(b). It appears that the bombardment of $\mathrm{LaO}^{-}$ions reduces both crystallite sizes and symmetry while simultaneously increases microstrains and unit cell volumes. Since the microstrains increase with increases of unit cell volume, it is naturally to assume that the negative pressure is induced by microstrains. This pressure may be derived from the Murnaghan's equation ${ }^{4,26}$, or by modelling the structures under stress using the Density Functional Theory (DFT). Figure 2(c) compares $\varepsilon$, translated into the equivalent volume expansions $\Delta V / V$, with calculated values obtained from DFT. In general, $\mathrm{LaMnO}_{3}$ is a challenging case for DFT modelling ${ }^{27}$ where Local Density Approximation (LDA) is known to provide good estimates of band-gap and structure, but more elaborated functionals (GGA) are also used ${ }^{28,29}$. To reproduce the band gaps ${ }^{30}$ and ground state correctly the on-site Coulomb repulsion ( $\mathrm{U}$ parameter) and antiferromagnetic spin configuration need to be included. For our cases, the best agreement is achieved with LDA functional for $\mathrm{U}=6.5 \mathrm{eV}$ (2.8\% error in band-gap, $0.9 \%$ in cell volume), whereas the deviations are large with GGA ( $>200 \%$ in band gap, $15 \%$ in cell volume). It is interesting to estimate how much external pressure is needed to force the same volume change as what is induced by increase of internal stress. Of course, the volume compression is not necessarily the same as the volume expansion, but we assume them to be equivalent. As seen, the dependence of $\Delta \mathrm{V} / \mathrm{V}$ on irradiation dose corresponds well to that of the simulated $\Delta \mathrm{V} / \mathrm{V}$ on external pressure. The agreement between the pressures derived from Murnaghan's equation and LDA is also good. Particularly, $8.0 \mathrm{GPa}$ increase of pressure stimulates a unit cell compression by $3.6 \%$ (while raises the ground state energy by $\sim 100 \mathrm{meV}$, Fig. 2(d), the relative increase is about $12.4 \mathrm{meV} / \mathrm{GPa}$ ), so it follows that $7.8 \mathrm{GPa}$ is needed to remove the microstrains related remaining pressure $(\sim 2.0 \mathrm{GPa}$, Fig. $2(\mathrm{c}))$ and compress the cell of $100 \mu \mathrm{C}$ irradiated sample into an equal cell of the un-doped one. These values are found also in good 
(a)

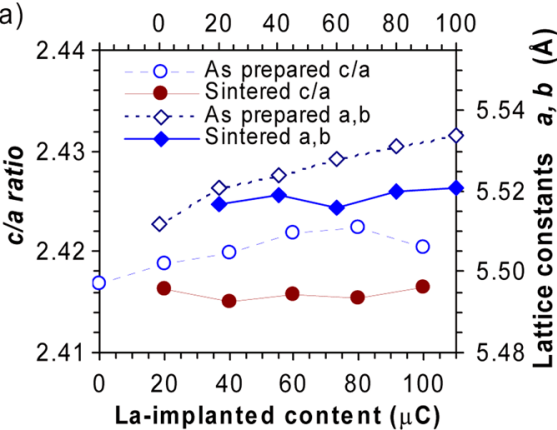

(c)

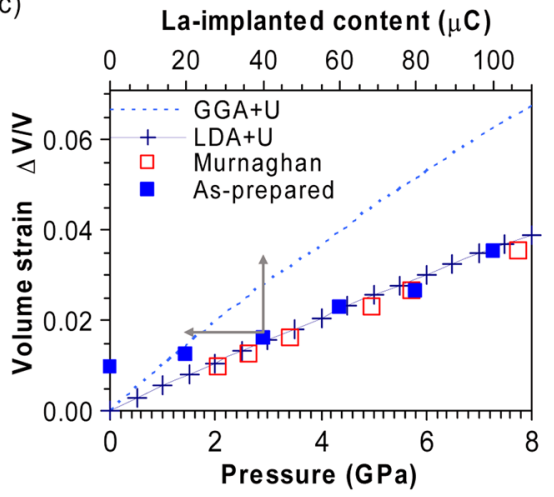

(b)

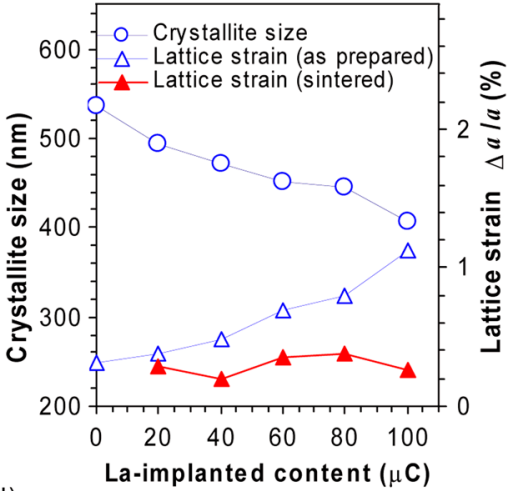

(d)

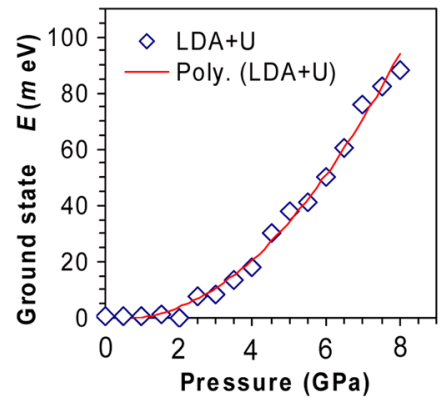

Figure 2. Lattice constants, crystallite sizes and strains of irradiated samples. (a) The lattice constants for asprepared and sintered samples; (b) The crystallite sizes and lattice strains; (c) LDA $+U$ calculated external pressure and Murnaghan's empirical pressure $\left(\Delta V / V=1-e^{-P / c}\right)^{26} ;$ (d) The increase of LDA+U ground state energy upon pressure (polynomial fitting is given).

agreement with the previous data reported for LMO under isotropic pressure ${ }^{4}$. Thus, the physics here is simple: the increase of dose leads to the increase of microstrains, which in turn increases the internal stress and the expansion of volume is a consequence.

Now to assist the analysis of Raman spectra, we discuss the effect of stress on positions of oxygen atoms. In $R \overline{3}$ $c$ the oxygen atoms occupy the Wyckoff's sites $18 e(x, 0,1 / 4)^{23}$ with $x \approx 0.43$. When $x=0.50$ the structure reduces to cubic, and for $x=0.40$ the $\mathrm{O}_{6}$ cages are strongly tilted, but still remain symmetric with 6 equal $\mathrm{Mn}-\mathrm{O}$ bonds. The only possible crystallographic invariant deformation is the precession rotation of $\mathrm{O}_{6}$ cages (Fig. 3(a)). The reduced rhombohedral cells are shown in Fig. 3(b), where preferred thermal motions of oxygen atoms around the rhombohedral axis $[101]_{r}$ are revealed by the shapes of thermal ellipsoids. As seen, two symmetry related $\mathrm{O}_{6}$ cages 1 and 2 possess different precessions and their rotation axes are parallel only in the cubic structure. The corresponding changes of $\mathrm{Mn}-\mathrm{O}$ distances and $\mathrm{Mn}-\mathrm{O}-\mathrm{Mn}$ angles are shown in Fig. 3(c), together with the theoretical values deduced directly from $x$. While the $\mathrm{Mn}-\mathrm{O}-\mathrm{Mn}$ angle (related to rotation of $\mathrm{O}_{6}$ cages) is practically linear with $x$ and is independent of cell choices, the $\mathrm{Mn}-\mathrm{O}$ distance (related to breathing of $\mathrm{O}_{6}$ cages) shifts to higher values for the sintered and as-prepared samples. The linear change of $\mathrm{Mn}-\mathrm{O}-\mathrm{Mn}$ angle upon rhombohedral distortion (inset, Fig. 3(c)) and the corresponding linearity of the soft modes' shifts upon pressure (Table 2) can be regarded as the good evidences for the equivalence of the actions of positive and negative pressure in low dose region.

Raman spectra of structures under stress. The obtained Raman scattering spectra are shown in Fig. 4 for the un-doped sample, the front sides (which face against the ion beam) of the as-prepared samples (Fig. 4(a)), the back sides (which attach to the substrate) of the same samples (Fig. 4(b)), and the sintered samples (front sides, Fig. 4(c)). The comparison of spectra obtained for $60 \mu \mathrm{C}$ irradiated sample is featured in Fig. 4(d). At the first sight, it appears that the scattering patterns of the fresh as-prepared samples are different for the front sides and the back sides. Also, the patterns from the back sides are similar to that of the un-doped sample. The similarity also appears between the un-doped and the sintered samples (Fig. 4(b)). The process of strains and relaxation is illustrated in Fig. 4(d) where one may see a split of a new peak at $558 \mathrm{~cm}^{-1}$ (front side) from a broadened peak at $450-600 \mathrm{~cm}^{-1}$ (back side). This split disappears for a sintered sample. The similarity between the un-doped and sintered samples again confirms on the absence of annealing effect caused by laser beam during Raman experiment. The obtained peaks positions are listed in Table 2. Two scattering bands at $544-563$ and $638-644 \mathrm{~cm}^{-1}$ show the largest blue shifts which are almost linear upon doses. For the un-doped sample, the peaks are identified at $326,486,544$ and $638 \mathrm{~cm}^{-1}$. The same peak structure is seen for the back sides of as-prepared samples, with some variation in frequencies: $320-328,486-489,551-556$, and $632-641 \mathrm{~cm}^{-1}$. The main deviations of the front sides from the others include $(i)$ clear splits of new peaks at $544-563 \mathrm{~cm}^{-1}$ and (ii) disappearances of shoulders at 
(a)

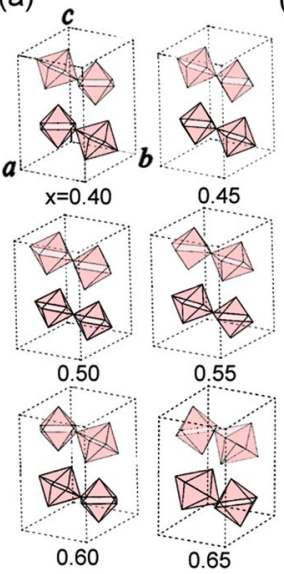

(b)

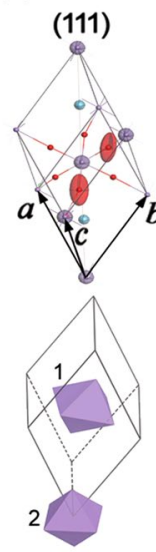

(c)

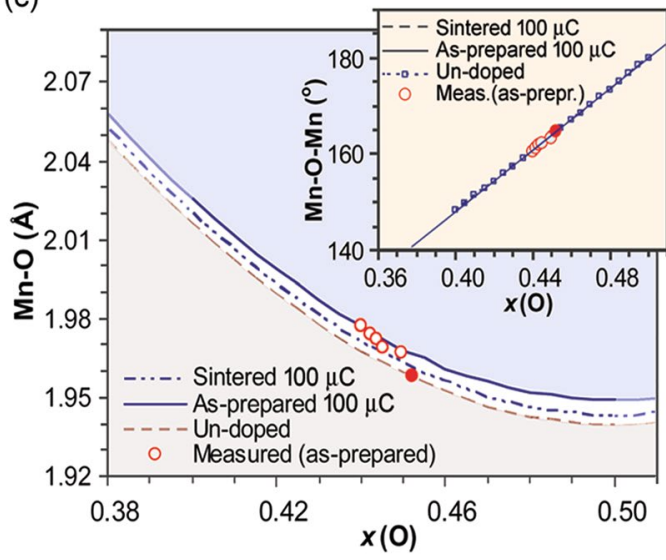

Figure 3. Illustration of rhombohedral distortion. (a) LMO in hexagonal representation: rotations of $\mathrm{MnO}_{6}$ octahedra as $x$-coordinates of oxygen atoms change; (b) LMO in a rhombohedral primitive cell: preferred movements by thermal ellipsoids, and stacking of tilted $\mathrm{MnO}_{6}$ octahedra; (c) Theoretical variations of $\mathrm{Mn}-\mathrm{O}$ distance and $\mathrm{Mn}-\mathrm{O}-\mathrm{Mn}$ angle (the inset) according to $x$-coordinates of oxygen for the sintered $(100 \mu \mathrm{C})$, asprepared $(100 \mu \mathrm{C})$ and un-doped cell. The measured data are also given for the as-prepared samples (red circles, the filled ones denote the un-doped case).

\begin{tabular}{|c|c|c|c|c|c|c|c|c|c|c|}
\hline \multirow[b]{2}{*}{ Assigned } & \multicolumn{5}{|c|}{ As-prepared (Front sides) } & \multicolumn{5}{|c|}{ As-prepared (Back sides) } \\
\hline & 20 & 40 & 60 & 80 & 100 & 20 & 40 & 60 & 80 & 100 \\
\hline $\mathrm{O}\left(A_{1 g}\right)$ & & & 257 & 256 & 260 & & & & & \\
\hline $\mathrm{O}_{6}\left(E_{g}\right)$ & 326 & 327 & 325 & 325 & 325 & 326 & 325 & 322 & 320 & 328 \\
\hline $\mathrm{O}_{6}\left(E_{g}\right)$ & 417 & 421 & 420 & 418 & 419 & & & & & \\
\hline $\mathrm{O}\left(E_{g}\right)$ & 486 & 486 & 484 & 488 & 489 & 486 & 488 & 486 & 488 & 489 \\
\hline $\mathrm{O}\left(E_{g}\right)$ & 557 & 556 & 558 & 559 & 563 & 551 & 552 & 554 & 556 & 553 \\
\hline $\mathrm{O}\left(E_{g}\right)$ & 640 & 639 & 641 & 643 & 644 & 641 & 636 & 632 & 639 & 635 \\
\hline $\mathrm{O}\left(E_{g}\right)$ & & 667 & & & & & 664 & 666 & 665 & 667 \\
\hline \multirow{3}{*}{ Un-doped } & \multicolumn{5}{|c|}{ Sintered samples (Front sides) } & \multicolumn{5}{|c|}{ Results of other works on $R \overline{3} c$ LMO } \\
\hline & 20 & 40 & 60 & 80 & 100 & ref. ${ }^{9}$ & 10 & 46 & 45 & 18 \\
\hline & & & & & & 236 & 230 & 210 & 217 & 205 \\
\hline \multirow[t]{2}{*}{326} & 327 & 327 & 328 & 328 & 325 & 329 & 315 & 313 & 323 & 320 \\
\hline & 414 & 411 & 417 & 414 & 407 & & 435 & 412 & 427 & 425 \\
\hline 486 & 488 & 487 & 490 & 490 & 487 & & 495 & 492 & 497 & 486 \\
\hline 544 & 550 & 541 & 547 & 557 & 539 & 520 & 505 & & & \\
\hline 638 & 644 & 634 & 632 & 630 & 642 & 640 & 620 & 605 & 618 & 610 \\
\hline 665 & & 662 & 665 & 663 & & & & & & \begin{tabular}{|l}
658 \\
\end{tabular} \\
\hline
\end{tabular}

Table 2. Peak positions $\left(\mathrm{cm}^{-1}\right)$ for the un-doped $R \overline{3} c \mathrm{LMO}$, the front sides and back sides of irradiated asprepared samples and the sintered samples. The doping contents are given in $\mu \mathrm{C}$.

around $665 \mathrm{~cm}^{-1}$. Two bands with minor intensities are seen at $256-260$ and $417-421 \mathrm{~cm}^{-1}$. The later is also repeated in the spectra of sintered samples $\left(407-417 \mathrm{~cm}^{-1}\right)$. Unlike in Pnma the Raman in $R \overline{3} c$ exhibit only a few active modes due to constrains in symmetry. According to group theor $\mathrm{y}^{31}$, the following active modes are available for each Wyckoff's sites:

$\mathrm{La}(6 \mathrm{a}): \mathrm{M}=\operatorname{Silent}\left(A_{2 g}\right)+\operatorname{IR}\left(A_{2 u}+E_{u}\right)+\operatorname{Raman}\left(E_{g}\right)$

$\operatorname{Mn}(6 \mathrm{~b}): \mathrm{M}=$ (Hyper-Raman) $A_{1 u}+\operatorname{IR}\left(A_{2 u}+2 E_{u}\right)$, (no Raman active mode)

$\mathrm{O}$ (18e): $\mathrm{M}=($ Hyper-Raman $) A_{1 u}+\operatorname{Silent}\left(2 A_{2 g}\right)+\operatorname{IR}\left(2 A_{2 u}+3 E_{u}\right)+\operatorname{Raman}\left(A_{1 g}+3 E_{g}\right)$

Therefore, a final mechanical representation of Raman contains only $1 A_{1 g}$ and $4 E_{g}$ modes. The previous lattice dynamic calculation ${ }^{9}$ revealed the $A_{1 g}$ at $249 \mathrm{~cm}^{-1}$ (assigned to $\mathrm{O}_{6}$ rotation around hexagonal [001] $]_{h}$ axis), one oxygen-related $E_{g}$ modes at 468 (bending vibration) and another at $646 \mathrm{~cm}^{-1}$ (out-of-phase stretching vibration). The assignment of $A_{1 g}$ mode to $\mathrm{O}_{6}$ rotation is consistent with our analysis of precession rotations of $\mathrm{O}_{6}$ cages (Fig. 3), so it seems to be unambiguous that $256-260 \mathrm{~cm}^{-1}$ peaks observed in 80 and $100 \mu \mathrm{C}$ as-prepared samples attribute to $A_{1 g}$ proper $R \overline{3} c$ mode. But it is better to describe it as precession rotations than pure rotations ${ }^{9}$. This peak is well reproduced from our calculated Phonon Density of States (PDOS) (Fig. 5), obtained by Lattice Dynamic Calculation (LDC) using the shell model (UNISOFT code ${ }^{32}$, with the same settings as of previous study $^{9}$ ). The PDOS for LMO has been studied experimentally by inelastic neutron scattering ${ }^{33}$ where many 

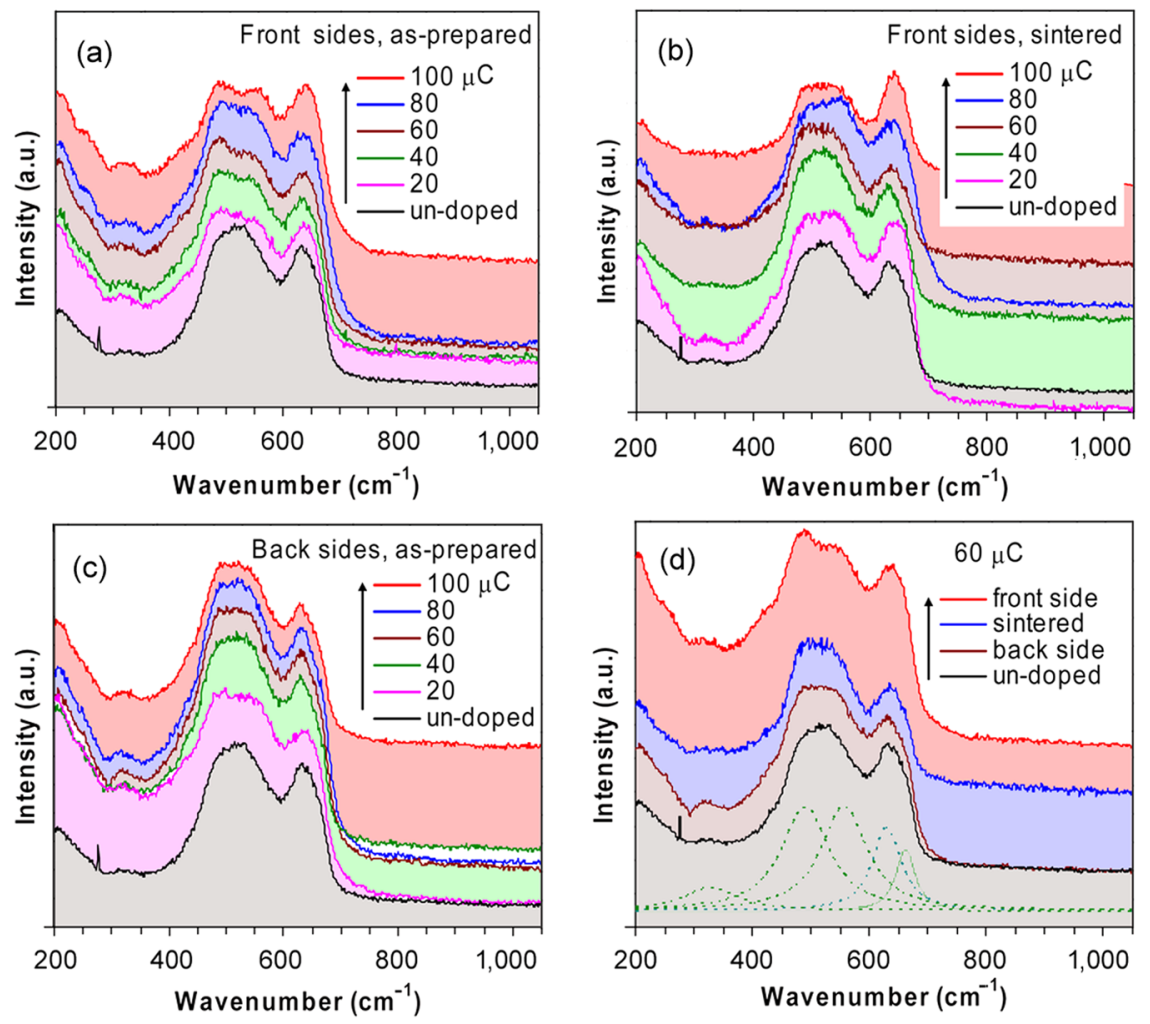

Figure 4. Raman spectra of polycrystalline LMO in $R \overline{3} c$ recorded at room temperature. (a) The front sides of asprepared samples; (b) The front sides of sintered samples; (c) The back sides of as-prepared samples; (d) Comparison of spectra recorded for one sample $(60 \mu \mathrm{C})$.

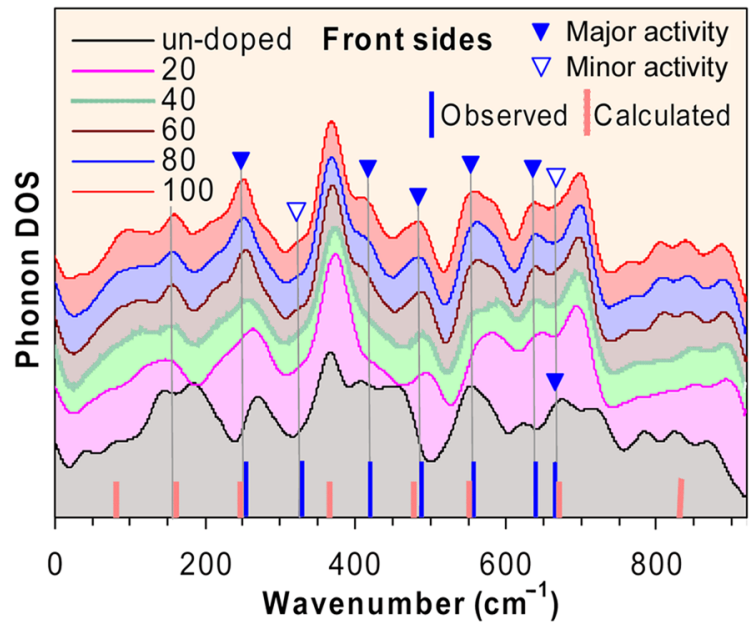

Figure 5. Calculated phonon density of states (PDOS) for implanted samples. Filled (blank) triangles denote major (minor) activities. Observed and calculated Raman activities in $R \overline{3} c$ are also shown.

overlaps with the PDOS given in Fig. 5 can be recognized. It appears that the peak at $256-260 \mathrm{~cm}^{-1}$ contributes to the main PDOS activities in 60, 80 and $100 \mu \mathrm{C}$ irradiated samples but reduces to sidebands in $20,40 \mu \mathrm{C}$ irradiated and un-doped samples. As discussed previously, the decrease of irradiation dose reduces microstrains and internal stress, which is equivalent to applying larger external pressure. This increase of external pressure at lower doses agrees well with the previous observations ${ }^{34}$ when this band diminishes at external pressures above 5.6 GPa. For our case of $40 \mu \mathrm{C}$, when this peak disappears, an internal pressure derived from Fig. 2(c) is $3.4 \mathrm{GPa}$. Taking into account that $7.8 \mathrm{GPa}$ is a corresponding internal pressure for $100 \mu \mathrm{C}$ sample, the $4.4 \mathrm{GPa}$ difference between the two cases is what we need to apply as an external pressure on $100 \mu \mathrm{C}$ sample to induce the disappearance of 256$260 \mathrm{~cm}^{-1}$ peak. It is commonly understood that, the increase of external pressure forces higher symmetry and reduces polarisation ${ }^{4}$. This is why the intensity of $256-260 \mathrm{~cm}^{-1}$ peak is small and diminishes at increasing external 
pressure (as $x$ is sliding towards 0.50 , Fig. 3(a,c)). This behaviour of a Raman mode is frequently referred to as belonging to rhombohedral distortion related soft modes ${ }^{9,35}$. The same point of view may be useful in explaining the intriguing activities found in Ca-doped $\mathrm{LMO}^{36}$. In this study, the two peaks at 235 and $435 \mathrm{~cm}^{-1}$ are expressing the blue shifts as temperature decreases. Because a thermal volume compression $\Delta \mathrm{V} / \mathrm{V} \approx 0.45 \%$ is expected for $200 \mathrm{~K}$ decrease of temperature, a corresponding external pressure $\approx 1.0 \mathrm{GPa}$ is deduced from Fig. 2 (c). So, the blue shifts in Ca-doped $\mathrm{LMO}^{36}$ may be explained in term of increasing oscillator strengths due to stress.

It follows from Fig. 5 that the peaks at $486-489 \mathrm{~cm}^{-1}$ also express the similarity to the ones at $256-260 \mathrm{~cm}^{-1}$. Their positions vary only a little and do not seem to depend on irradiation dose, while their activities dominate in 60,80 and $100 \mu \mathrm{C}$ samples but decrease for $20,40 \mu \mathrm{C}$ and the un-doped ones. The same activities are also exhibited by the peaks at $638-644 \mathrm{~cm}^{-1}$, but with clear blue shifts on increasing dose. Recall that the similar peaks were identified as $E_{g}$ pure oxygen bending vibrations $\left(510 \mathrm{~cm}^{-1}\right)^{9}$ and $E_{\mathrm{g}}$ out-of-phase stretching oxygen vibration $\left(640 \mathrm{~cm}^{-1}\right)^{9}$. Since the intensities of these peaks are strong, they are often observed in a number of cases (Table 2). They may also disappear under certain circumstances, i.e. below Curie temperature $T_{c}^{10}$, above Jahn-Teller transition temperature $T_{\mathrm{JT}}(\sim 710 \mathrm{~K})^{11}$, under magnetic field ${ }^{37}$, or in strongly doped cases ${ }^{17,38}$. Both $486-489$ and $638-$ $644 \mathrm{~cm}^{-1}$ bands couple with Jahn-Teller distortion of $\mathrm{O}_{6}$ cages $^{13}$ but the assigned oxygen vibrations are still in question $^{8,39}$. It is commonly agreed that both are one phonon modes, attributed to elementary excitation caused by orbital ordering (alias orbitons), or orbitons coupled with phonons ${ }^{16,21,40}$. The blue shifts of both modes are reported for $R \overline{3} c \mathrm{LMO}$ thin films ${ }^{20,41}$. The shifts seen in our samples for $638-644 \mathrm{~cm}^{-1}$ peaks can be regarded as the blue shifts at the volume expansions. Intuitively, the increase of external pressure increases Mn-O force constant $\kappa$, and proper Mn-O vibration frequency $\nu(\nu=\sqrt{\kappa / \mu} / 2 \pi, \mu$ is a reduced mass). This consequently leads to blue shifts at volume compression, not at expansion as in our cases. Indeed, the pressure dependent blue shifts are often reported ${ }^{34}$. They occur even in cases where external pressure is not explicitly present, as for the oxygen excess compounds $\mathrm{LaMnO}_{3+\delta}$ (Fig. 1 therein) $)^{21}$ : the systematic blue shifts from 605 to $615 \mathrm{~cm}^{-1}$ are observed when $\delta$ increases from 0.0 to 0.07 . This is because in the isothermal regime the pressure must increase when the volume compresses according to increasing $\delta$. So far, the red shifts observed in our cases do not fall into this scheme. Naturally, it leads to the non-uniform impacts of microstrains, and argues for the fact that the microstrains may locally impose both volume expansion and compression. We can expect that the two effects are canceling each other macroscopically but at the microscopic level, where Raman is active, a small imbalance in favor of compression may induce blue shifts. So we can observe the blue shifts even in overall volume expansions. The data from our analysis confirm this scenario: the TCH strains $\varepsilon_{\theta}$ are twice as large as the lattice strains $\varepsilon_{a}$, so the local compression of volume may be twice larger than the global average volume expansion.

As also depicted in Fig. 5, the peaks observed in a range $544-563 \mathrm{~cm}^{-1}$ (that split from a broad feature around $500 \mathrm{~cm}^{-1}$ ) behave nearly in the same manner as the ones at $638-644 \mathrm{~cm}^{-1}$. These peaks were not usually resoluble in the previous studies ${ }^{9,10,19,20}$ but the similar features are seen in the lower Pnma space group, such as in $\mathrm{La}_{1-\mathrm{x}} \mathrm{Ca}_{\mathrm{x}} \mathrm{MnO}_{3-\delta}\left(555 \mathrm{~cm}^{-1}, B_{3 \mathrm{~g}}(2)\right.$ out-of-phase bending $\left.{ }^{17}\right), \mathrm{CaMnO}_{3}\left(564 \mathrm{~cm}^{-1}, B_{3 \mathrm{~g}}(2)\right.$ symmetric breathing ${ }^{42}$, $\mathrm{La}_{0.7} \mathrm{Sr}_{0.3} \mathrm{MnO}_{3}\left(562 \mathrm{~cm}^{-1}, A_{\mathrm{g}}\right.$ in-phase stretching $\left.{ }^{43}\right)$. The absence of these peaks in higher symmetry (or at high pressure $^{34}$ ) directly links them to stress: the disappearance at lower doses is caused by the decrease of polarisation as symmetry increases. So, we suggest that the significant blue shifts of $544-563 \mathrm{~cm}^{-1}$ bands at increasing irradiation doses may also be explained by the non-uniform impacts of microstrains. A result of linear fitting yields $\nu\left(\mathrm{cm}^{-1}\right)=569.2-2.413 \times P$ (with $P$ is pressure in GPa). A slope of this equation agrees quite well with the ones retrieved from the data previously published ${ }^{34}$ for the same peaks. Since these peaks are clearly resoluble only when dislocations caused by irradiation increases, they should be assigned to a forbidden mode which is related to anti-symmetric vibrations of $\mathrm{O}_{6}$ cages.

Another scattering feature directly related to stress is a small peak within $662-667 \mathrm{~cm}^{-1}$ that is observed only in a few cases (Table 2). This peak corresponds to minor activity in PDOS of all irradiated samples but is a main activity in the un-doped one (Fig. 5). A similar feature has been reported previously for LMO under external pressure: above $3.0 \mathrm{GPa}^{34}$ (or $7.0 \mathrm{GPa}^{4}$ ) there appears a new band at around $680 \mathrm{~cm}^{-1}$ whose intensity grows with increasing pressure. This behaviour corresponds very well to the disappearance of a shoulder peak at around $665 \mathrm{~cm}^{-1}$ in our spectra, this peak cannot be seen in the spectra of 60,80 , and $100 \mu \mathrm{C}$ as-prepared samples (front sides) (Fig. 4(d)) but is presented quite clear in the un-doped sample, $40 \mu \mathrm{C}$ as-prepared and some sintered samples (front sides) and most back sides of as-prepared samples. As $3.0 \mathrm{GPa}$ is equal to a difference between the derived pressures for 100 and $60 \mu \mathrm{C}$ samples (Fig. 2(c)), the samples occur at lower doses (therefore at higher external pressures than $3.0 \mathrm{GPa}$ ) are approaching the occurrence of this new resonance band. According to the previous studies ${ }^{4,34}$ this peak is caused by the co-existence of undistorted octahedra (with $x=0.5$ ) that are formed at high pressure. The occurrence of undistorted octahedra is necessarily associated with local changes of symmetry of connecting oxygen sites between two neighbouring $\mathrm{MnO}_{6}$ octahedra. This situation is similar to that of $\mathrm{YMnO}_{3}$ in $\mathrm{P}_{3} \mathrm{~cm}$ space group, where two apical oxygen atoms possess different site symmetry than that of the rest, so their displacements along axis $z$ were assigned to a most pronounced resonance band observed at $681 \mathrm{~cm}^{-1}$ in $\mathrm{YMnO}_{3}{ }^{44}$. For $\mathrm{LMO}$, a peak close to our $662-667 \mathrm{~cm}^{-1}$ has been reported ${ }^{13}$ at $655 \mathrm{~cm}^{-1}$ when the excitation wavelengths were shorter than $351 \mathrm{~nm}$ (energy greater than $3.53 \mathrm{eV}$ ) and was interpreted as a parent one phonon mode of a two phonon seen at $1300 \mathrm{~cm}^{-1}$. It couples strongly with a charge-transfer gap at $4.4 \mathrm{eV}$ and is assigned to a vibrational oxygen breathing mode ${ }^{13}$. A peak at around $670 \mathrm{~cm}^{-1}$, whose intensity depends strongly on laser focus region, is also reported for rhombohedral La deficit structure $\mathrm{La}_{0.91-\delta} \mathrm{Mn}_{1-\delta} \mathrm{O}_{3}{ }^{10}$. Interestingly, this peak can also be seen in the orthorhombic La deficit $\mathrm{La}_{1-\delta} \mathrm{Mn}_{1-\delta} \mathrm{O}_{3}$ compounds. So its presence does not seem to depend on space group symmetry but rather relates to local deformations associated with one of oxygen sites and couples with uni-axial motions of this site along one octahedral axis (e.g. a rhombohedral axis [101 $\left.]_{r}\right)$. Therefore, the symmetric oxygen breathing mode appears as a reasonable assignment for the $662-667 \mathrm{~cm}^{-1}$ shoulders. The symmetry also explains why they become stronger when symmetry increases in high pressure. 
Lastly, we discuss the two weak features observed in most of our samples, to which the previous studies seem not to pay enough attentions: the clear peaks at $320-328 \mathrm{~cm}^{-1}$ and the minor shoulders at $407-419 \mathrm{~cm}^{-1}(\mathrm{missing}$ for the back sides). For comparison, we remind that, peaks in the same frequency range $\left(320-328 \mathrm{~cm}^{-1}\right)$ are reported for Pnma LMO (identified as $B_{3 g}$ in-phase $z$ rotation mode) ${ }^{8}, R \overline{3} c$ LMO ( $E_{g}$ pure Mn vibration mode $)^{9,18,45}$, and for both orthorhombic and rhombohedral La deficit $\mathrm{LMO}^{10}$. The peaks at lower frequencies (but close to $320 \mathrm{~cm}^{-1}$ ) are seen in some doped cases, e.g. in the $\mathrm{Na}$-doped $\mathrm{LMO}^{46}$. On the other hand, a peak treated as rotational mode ${ }^{13}$ is presented at $448 \mathrm{~cm}^{-1}$. A peak assigned to an $E_{g}$ mode also appear for Na-doped $\mathrm{LMO}^{46}$ at $436.6 \mathrm{~cm}^{-1}$, for $\mathrm{Sr}, \mathrm{Zn}$-doped $\mathrm{LMO}^{35}$ at $427 \mathrm{~cm}^{-1}$. For both bulk and thin films $\mathrm{LMO}^{45}$ the similar peaks can be observed at around $425 \mathrm{~cm}^{-1}$. The La deficit LMOs represent the cases where peaks can be found at $420 \mathrm{~cm}^{-1}$ in both orthorhombic and rhombohedral samples ${ }^{10}$. Since both features in our cases, $320-328$ and $417-421 \mathrm{~cm}^{-1}$, show no clear dependence on irradiation, it will be naturally to suppose that these peaks are not related to rhombohedral distortions of $\mathrm{O}_{6}$ cages but to other kinds of displacements of atoms. Indeed, the results from our simulation show that there is a uni-axial translation-like movement of fixed $\mathrm{O}_{6}$ cages. There are two main directions, one is along a line bisecting two neighbouring $\mathrm{O} \ldots \mathrm{O}$ atoms $\left(310-330 \mathrm{~cm}^{-1}\right)$ and another is along one $\mathrm{Mn}-\mathrm{O}$ bond $\left(400-425 \mathrm{~cm}^{-1}\right)$. A circular polarisation of La atoms may contribute to both these resonances too. Such kind of modes, of course should not be changed among the compounds, and indeed they are observed in different samples and symmetries.

\section{Methods}

Preparation of bulk materials. The LMO bulk samples were prepared by using a sol-gel citrate method with nitrate salts of lanthanum and manganese $\left(0.5 \mathrm{M} \mathrm{La}\left(\mathrm{NO}_{3}\right)_{3}\right.$ and $0.5 \mathrm{M} \mathrm{Mn}\left(\mathrm{NO}_{3}\right)_{2}$ solutions $)$ as initial precursors. The stirred mixtures of equal amounts from each of these solutions were heated at $70^{\circ} \mathrm{C}$, and in continuous stirring the citric acid ( $1 \mathrm{M}$ solution) was slowly added to maintain the $\mathrm{pH}$ between 3 and 5 , until the white gels appeared. After drying, the gels were pre-sintered at $500^{\circ} \mathrm{C}$ for 4 hours, and the obtained dark powder was ground in open air, and pressed into the disks of diameter 10 and thickness $1 \mathrm{~mm}$ under a pressure of $4500 \mathrm{~kg} / \mathrm{cm}^{2}$. The final product was sintered at $1200^{\circ} \mathrm{C}$ for 40 hours in Ar atmosphere, resulting in the un-doped bulk LMO samples.

Irradiation by heavy-ions. The irradiated samples were obtained by subjecting the raw LMO disks to the $\mathrm{LaO}^{-}$ion beam, produced from a Cesium Enhanced Negative Ion Sputter Source (SNICS II) and accelerated to desired energy by an electrostatic accelerator (Pelletron 5SDH-2). The ion beam energy was adjusted to allow a penetration depth of around $100 \mu \mathrm{m}$ into the bulk targets. The total charges the beam imposed on the samples are $0,20,40,60,80$, and $100 \mu \mathrm{C}$, which imply the real bulk concentrations of less than $0.05 \%$. While the real distribution of ions in the whole sample is unknown and probably differs from case to case, we can safely consider at the first estimate that the distribution is Gaussian upon the implantation depth. This implies for our case of the thick discs that at the thin surface layers (where techniques like X-ray and Raman are active) the concentration is constant and the distribution is homogeneous in average. Recall that the accelerator in use is a Van de Graaff type accelerator, where the energy $E$ (in $\mathrm{MeV}$ ) of accelerated ions can be estimated empirically from the terminal voltage $V_{\pi}$ (in MV) and charge $q$ of ions as $E=\mathrm{V}_{\pi}(q+1)$. Therefore, the $\mathrm{LaO}^{-}$ions at maximum $1.7 \mathrm{MV}$ will attain maximum output energy of $3.4 \mathrm{MeV}$, which practically allows them to penetrate through thick layers of bulk materials. Thus, this type of accelerator is suitable for doping the bulk samples, unlike the early types which are equipped with low energy beams and can be used only for doping surfaces or thin films ${ }^{47}$.

Characterization measurements. The X-ray diffractograms were obtained on Bruker D5005 diffractometer with $\mathrm{CuK}_{\alpha}$ radiation $(\lambda=1.54056 \AA)$, for $2 \theta$ from 20 to $80^{\circ}$ at a step width of $0.03^{\circ}$. The Raman scattering measurements were performed at room temperature in backscattering geometry by using a He-Ne excitation laser of wavelength $632.8 \mathrm{~nm}$. For each sample the X-ray and Raman scattering spectra were recorded for the front sides, which face towards the ion beam, and the back sides, which are attached to the substrate. Furthermore, to examine the structural changes after implantation (also to rule out the possible laser annealing effect during Raman experiments) we annealed all samples again at $500^{\circ} \mathrm{C}$ for 8 hours in Ar and recorded the X-Ray and Raman spectra correspondingly.

DFT modeling. The calculation was processed with Local Density Approximation (LDA) of the following settings (CASTEP code $^{48}$ ): spin-polarized configuration, energy cut-off $380 \mathrm{eV}$, $k$-point set $3 \times 3 \times 2$ (Monkhorst-Pack grid), convergence $5.0 \times 10^{-7} \mathrm{eV} /$ atom, ultra-soft potential, plane wave basis with LDA $+\mathrm{U}$ correction $(\mathrm{U}=6.5 \mathrm{eV})$ for $\mathrm{Mn} d$-electrons. Only diagonal elements of stress tensor are selected and non-zero. The starting model structure is considered as stress free. The symmetry is $R \overline{3}$ with antiferromagnetic spin configuration.

\section{Data availability}

X-ray, Raman and DFT data are available upon request. Correspondences should be addressed to HNN.

Received: 14 September 2019; Accepted: 28 November 2019;

Published online: 16 December 2019

\section{References}

1. Baldini, M. et al. Origin of colossal magnetoresistance in $\mathrm{LaMnO}_{3}$ manganite. PNAS 112(35), 10869-10872 (2015).

2. Murakami, Y. et al. Resonant X-Ray Scattering from Orbital Ordering in LaMnO . Phys. Rev. Lett. 81(3), 582 (1998).

3. Pavarini, E. \& Koch, E. Origin of Jahn-Teller Distortion and Orbital Order in LaMnO $\mathrm{L}_{3}$ Phys. Rev. Lett. 104, 086402 (2010).

4. Loa, I. et al. Pressure-Induced Quenching of the Jahn-Teller Distortion and Insulator-to-Metal Transition in LaMnO $\mathrm{La}_{3}$. Phys. Rev. Lett. 87, 125501 (2001) 
5. Roqueta, J. et al. Strain-Engineered Ferromagnetism in $\mathrm{LaMnO}_{3}$ Thin Films. Cryst. Growth Des. 15(11), 5332-5337 (2015).

6. Zener, C. Interaction between the d-Shells in the Transition Metals. II. Ferromagnetic Compounds of Manganese with Perovskite Structure. Phys. Rev. 82(3), 403 (1951).

7. Inoue, J. et al. Raman scattering by orbital waves in perovskite $\mathrm{LaMnO}_{3}$. Physica B 237-238, 51-53 (1997).

8. Iliev, M. N. et al. Raman spectroscopy of orthorhombic perovskitelike $\mathrm{YMnO}_{3}$ and $\mathrm{LaMnO}_{3}$. Phys. Rev. B 57(5), 2872-2877 (1998).

9. Abrashev, M. V. et al. Comparative study of optical phonons in the rhombohedrally distorted perovskites $\mathrm{LaAlO}_{3}$ and $\mathrm{LaMnO}_{3}$. Phys. Rev. B 59(6), 4146-4153 (1999).

10. Granado, E. et al. Effects of Cation Vacancies in the Phonon Raman Spectra of $\mathrm{LaMnO}_{3}$. Phys. Stat. Sol. (b) 220, 609 (2000).

11. Granado, E., Sanjurjo, J. A., Rettori, C., Neumeier, J. J. \& Oseroff, S. B. Order-disorder in the Jahn-Teller transition of $\mathrm{LaMnO}_{3}: \mathrm{A}$ Raman scattering study. Phys. Rev. B 62(17), 11304 (2000).

12. Saitoh, E. et al. Observation of orbital waves as elementary excitations in a solid. Nature 410, 180 (2001).

13. Krüger, R. et al. Orbital ordering in $\mathrm{LaMnO}_{3}$ Investigated by Resonance Raman Spectroscopy. Phys. Rev. Lett. 92,097203 (2004).

14. Perebeinos, V. \& Allen, P. B. Multiphonon resonant Raman scattering predicted in $\mathrm{LaMnO}_{3}$ from the Franck-Condon process via self-trapped excitons. Phys. Rev. B 64, 085118 (2001).

15. Sugai, S. \& Hirota, K. Orbital waves in $\mathrm{YVO}_{3}$ studied by Raman scattering. Phys. Rev. B 73, 020409(R) (2006).

16. Munkhbaatar, P. \& Myung-Whun, K. Orbital wave in the Raman scattering cross section of $\mathrm{LaMnO}_{3}$. Phys. Rev. B 97, 085101 (2018).

17. Trotsenko, V. G., Lahmar, A., Lyanguzov, N. V., El Marssi, M. \& Torgashev, V. I. Phase separation and local lattice distortions analysis of charge-ordered manganese films $\mathrm{La}_{1-\mathrm{x}} \mathrm{Ca}_{\mathrm{x}} \mathrm{MnO}_{3-\delta}$ by Raman spectroscopy. Superlatt. \& Microstruc. 127, 100-108 (2019).

18. Das, P. T., Singh, R., Das, A. \& Nath, T. K. Structural, magnetic, and physical properties of $\mathrm{La}_{(1-\mathrm{x})} \mathrm{MnO}_{3 \pm \delta}$ nano-manganite. Phil. Magz. 96(3), 286-300 (2016).

19. Zhang, X., Zhang, A. M., Xie, W. M., Lin, J. G. \& Wu, X. S. Effect of strain-modulated lattice distortion on the magnetic properties of $\mathrm{LaMnO}_{3}$ films. Phys. B 476, 114-117 (2015).

20. Dubey, A. \& Sathe, V. G. The effect of magnetic order and thickness in the Raman spectra of oriented thin films of $\mathrm{LaMnO}_{3}$. J. Phys. Condens. Matter. 19, 346232 (2007).

21. Granado, E. et al. Magnetic ordering effects in the Raman spectra of $\mathrm{La}_{1-\mathrm{x}} \mathrm{Mn}_{1-\mathrm{x}} \mathrm{O}_{3}$. Phys. Rev. B 60(17), 11879 (1999).

22. Thompson, P., Cox, D. E. \& Hastings, J. B. Rietveld Refinement of Debye-Scherrer Synchrotron X-ray Data from $\mathrm{Al}_{2} \mathrm{O}_{3}$. J. Appl. Cryst. 20, 79-83 (1987).

23. Huang, Q. et al. Structure and magnetic order in undoped lanthanum manganite. Phys. Rev. B 55(22), 14987 (1997).

24. The Rietveld Method (ed. Young, R. A.), IUCr Monographs on Crystallography 5 (Oxford University Press, 1993)

25. Solanki, A. et al. Irradiation-induced modifications and PEC response - A case study of $\mathrm{SrTiO}_{3}$ thin films irradiated by $120 \mathrm{MeV}$ $\mathrm{Ag}^{9+}$ ions. Int'l. J. of Hydrogen Energ. 36, 5236-5245 (2011).

26. Murnaghan, F. D. The compressibility of media under extreme pressures. PNAS 30(9), 244-247 (1944)

27. Varignon, J., Bibes, M. \& Zunger, A. Origin of band gaps in 3d perovskite oxides. Nature Comm. 10, 1658 (2019).

28. Wang, Y. \& Cheng, H. P. Oxygen Reduction Activity on Perovskite Oxide Surfaces: A Comparative First-Principles Study of $\mathrm{LaMnO}_{3}, \mathrm{LaFeO}_{3}$, and $\mathrm{LaCrO}_{3}$. J. Phys. Chem. C 117(5), 2106-2112 (2013).

29. Nguyen, T. T. et al. Magnetic state of the bulk, surface and nanoclusters of $\mathrm{CaMnO}_{3}$ : A DFT study. Physica B 406, 3613-3621 (2011).

30. Arima, T., Tokura, Y. \& Torrance, J. B. Variation of optical gaps in perovskite-type 3d transition-metal oxides. Phys. Rev. B 48(23), $17006(1993)$.

31. Altmann, S. L. \& Herzig, P. Point-Group Theory Tables (Oxford Science Publications, 1994).

32. Eckold, G., Stein-Arsic, M. \& Weber, H. J. UNISOFT - a program package for lattice-dynamical calculations. J. Appl. Cryst. 20, 134-139 (1987).

33. Wdowik, U. D., Koza, M. M. \& Chatterji, T. Phonons in lanthanum manganite: Inelastic neutron scattering and density functional theory studies. Phys. Rev. B 86, 174305 (2012).

34. Baldini, M., Struzhkin, V. V., Goncharov, A. F., Postorino, P. \& Mao, W. L. Persistence of Jahn-Teller Distortion up to the Insulator to Metal Transition in $\mathrm{LaMnO}_{3}$. Phys. Rev. Lett. 106, 066402 (2011).

35. Dubroka, A. et al. Raman and infrared studies of $\mathrm{La}_{1-y} \mathrm{Sr}_{\mathrm{y}} \mathrm{Mn}_{1-\mathrm{x}} \mathrm{M}_{\mathrm{x}} \mathrm{O}_{3}(\mathrm{M}=\mathrm{Cr}, \mathrm{Co}, \mathrm{Cu}, \mathrm{Zn}, \mathrm{Sc}$ or Ga): Oxygen disorder and local vibrational modes. Phys. Rev. B 73, 224401 (2006).

36. Irwin, J. C., Chrzanowski, J. \& Franck, J. P. Oxygen isotope effect on the vibrational modes of $\mathrm{La}_{1-\mathrm{x}} \mathrm{Ca}_{\mathrm{x}} \mathrm{MnO}_{3}$. Phys. Rev. B 59(14), 9362 (1999).

37. Li, J. M., Huan, C. H. A., Du, Y.-W., Feng, D. \& Shen, Z. X. Magnetic-field-tunable charge carrier localization in sintered polycrystalline $\mathrm{La}_{0.75} \mathrm{Ca}_{0.25} \mathrm{MnO}_{3}$. Phys. Rev. B 63, 024416 (2000).

38. Iliev, M. N., Abrashev, M. V., Popov, V. N. \& Hadjiev, V. G. Role of Jahn-Teller disorder in Raman scattering of mixed-valence manganites. Phys. Rev. B 67, 212301 (2003).

39. Kovaleva, N. N. et al. Anomalous multi-order Raman scattering in $\mathrm{LaMnO}_{3}$ : a signature of quantum lattice effects in a Jahn-Teller crystal. J. Phys. Condens. Matter. 25, 155602 (2013).

40. Xu, J., Park, J. H. \& Jang, H. M. Orbital-spin-phonon coupling in Jahn-Teller-distorted $\mathrm{LaMnO}_{3}$ : Softening of the 490 and $610 \mathrm{~cm}^{-1}$ Raman-active modes. Phys. Rev. B 75, 012409 (2007).

41. Chaturvedi, A. \& Sathe, V. Thickness dependent Raman study of epitaxial $\mathrm{LaMnO}_{3}$ thin films. Thin Sol. Film. 548, 75-80 (2013).

42. Abrashev, M. V. et al. Raman spectroscopy of $\mathrm{CaMnO}_{3}$ : Mode assignment and relationship between Raman line intensities and structural distortions. Phys. Rev. B 65, 184301 (2002).

43. Behera, B. C., Ravindra, A. V., Padhan, P. \& Prellier, W. Raman spectra and magnetization of all-ferromagnetic superlattices grown on (110) oriented $\mathrm{SrTiO}_{3}$. Appl. Phys. Lett. 104, 092406 (2014).

44. Iliev, M. N. et al. Raman- and infrared-active phonons in hexagonal $\mathrm{YMnO}_{3}$ : Experiment and lattice-dynamical calculations. Phys. Rev. B 56(5), 2488-2494 (1997).

45. Khanduri, H. et al. Magnetic and structural studies of $\mathrm{LaMnO}_{3}$ thin films prepared by atomic layer deposition. J. Phys. D: Appl. Phys. 46, 175003 (2013).

46. Dodiya, N. \& Varshney, D. Structural properties and Raman spectroscopy of rhombohedral $\mathrm{La}_{1-\mathrm{x}} \mathrm{Na}_{\mathrm{x}} \mathrm{MnO}_{3}(0.075 \leq \times \leq 0.15)$. J. Mol. Struc. 1031, 104-109 (2013).

47. Jain, I. P. \& Agarwal, G. Ion beam induced surface and interface engineering. Surf. Sci. Rep. 66, 77-172 (2011).

48. Clark, S. J. et al. First principles methods using CASTEP. Zeitschrift für Kristallographie 220(5-6), 567-570 (2005).

\section{Acknowledgements}

This research is funded by Vietnam National Foundation for Science and Technology Development (NAFOSTED) under the Grant Number 103.02-2017.18.

\section{Author contributions}

H.N.N. conceived the ideas, designed the experiment, analysed data, and wrote the manuscript. P.D.H.Y. performed the preparation of materials, X-ray and Raman measurements. N.T.N. performed the ion beam irradiation experiments. 


\section{Competing interests}

The authors declare no competing interests.

\section{Additional information}

Correspondence and requests for materials should be addressed to N.N.H.

Reprints and permissions information is available at www.nature.com/reprints.

Publisher's note Springer Nature remains neutral with regard to jurisdictional claims in published maps and institutional affiliations.

(c) (i) Open Access This article is licensed under a Creative Commons Attribution 4.0 International License, which permits use, sharing, adaptation, distribution and reproduction in any medium or format, as long as you give appropriate credit to the original author(s) and the source, provide a link to the Creative Commons license, and indicate if changes were made. The images or other third party material in this article are included in the article's Creative Commons license, unless indicated otherwise in a credit line to the material. If material is not included in the article's Creative Commons license and your intended use is not permitted by statutory regulation or exceeds the permitted use, you will need to obtain permission directly from the copyright holder. To view a copy of this license, visit http://creativecommons.org/licenses/by/4.0/.

(c) The Author(s) 2019 